\title{
Lymphoepithelioma-Like Carcinoma of the Skin Treated with Wide Local Excision and Chemoradiation Therapy: A Case Report and Review of the Literature
}

\author{
Theresa M. Gille, ${ }^{1}$ Edward F. Miles, ${ }^{2}$ and Allen O. Mitchell ${ }^{1}$ \\ ${ }^{1}$ Department of Otolaryngology-Head and Neck Surgery, Naval Medical Center Portsmouth, 620 John Paul Jones Circle, \\ Portsmouth, VA 23708, USA \\ ${ }^{2}$ Division of Radiation Oncology, Department of Radiology, Naval Medical Center Portsmouth, 620 John Paul Jones Circle, \\ Portsmouth, VA 23708, USA \\ Correspondence should be addressed to Edward F. Miles, edward.miles@med.navy.mil
}

Received 26 July 2012; Accepted 10 October 2012

Academic Editors: A. Goodman, E. Itakura, J. I. Mayordomo, and Y. Yokoyama

Copyright (๑) 2012 Theresa M. Gille et al. This is an open access article distributed under the Creative Commons Attribution License, which permits unrestricted use, distribution, and reproduction in any medium, provided the original work is properly cited.

Lymphoepithelioma-like carcinoma of the skin (LELCS) is a rare cutaneous neoplasm microscopically similar to undifferentiated nasopharyngeal carcinoma. It is typically nonaggressive and is treated with wide local excision. However, we present a case of a patient with a regional recurrence and more aggressive LELCS with perineural invasion and positive margins for which he was treated with wide local excision followed by chemoradiation. We discuss the use of chemoradiation for this patient and review the literature, specifically pertaining to treatment of more aggressive cases of LELCS.

\section{Introduction}

Lymphoepithelioma-like carcinoma of the skin (LELCS) is an uncommon primary cutaneous neoplasm with less than 60 cases reported in the literature since it was first described by Swanson et al. in 1988 [1]. It has extremely low metastatic potential. Surgical excision is the preferred treatment although radiation has been used in cases which experienced a local recurrence or metastatic disease [1$3]$. The use of radiotherapy is currently not well described for LELCS particularly in cases where there were positive margins or perineural invasion. We report a case of a gentleman with a regional recurrence and more aggressive LELCS showing perineural invasion and positive margins after excision. Due to the more aggressive nature of the tumor he was treated with chemoradiation postoperatively.

\section{Case Report}

A 76-year-old male presented to our clinic with a severalmonth history of a pea-sized lesion in his left upper lip which had been resected by another physician and found to be LELCS. The pathology report for the initial excisional biopsy resection did not comment on margin status. The case was presented to the institution's multidisciplinary tumor board and it was decided that close observation was warranted, rather than further treatment. Ten months later the patient returned with a similar lesion in his right cheek. Physical exam revealed a firm area superolateral to the nasolabial fold on the contralateral side of the face measuring $1.5 \mathrm{~cm}$ in largest dimension. It was slightly mobile with respect to the maxilla and overlying skin. He had no palpable lymphadenopathy and there was no palpable lesion in the left upper lip at the site of the original resection. A computed tomography (CT) scan showed a $2.8 \times 2.6 \mathrm{~cm}$ soft tissue mass in the right premaxillary tissue and a positron emission tomography (PET)/CT scan showed increased metabolic activity in this tissue but not in the original site. In addition, the PET/CT also demonstrated a $2.2 \mathrm{~cm} \times 2.4 \mathrm{~cm}$ ground glass opacity in the left lower lobe of the lung which also had increased metabolic activity. A CT-guided fine needle aspiration of the lung lesion was performed which showed 


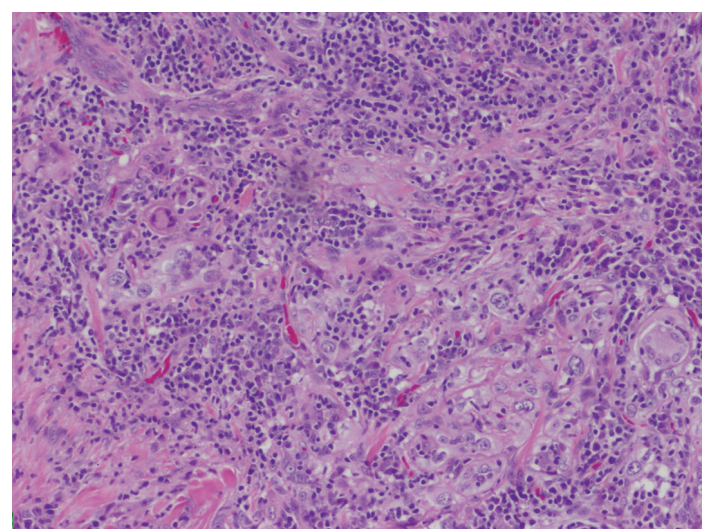

FIGURE 1: Hematoxylin and eosin stain (100x) showed aggregates of atypical epithelial cells and associated lymphoid tissue supported by fibrous connective tissue.

malignant cells consistent with nonsmall cell lung carcinoma (insufficient tissue was recovered for further pathologic delineation). Staging studies showed this to be a T1a, N0, M0, Stage IA lung cancer. The patient underwent surgical excision of the new lesion in his right premaxillary space. The pathology revealed LELCS (Figure 1) with infraorbital nerve invasion and vascular invasion as well as positive superficial, medial and deep margins and an indeterminant lateral margin. Immunohistochemical staining was positive for pancytokeratin and CK-5/6 (Figure 2). Additional infra-orbital nerve was resected and was negative for tumor involvement. The patient was again discussed at our institution's tumor board and it was decided that, due to the recurrent nature of this disease, the more aggressive phenotype demonstrating perineural invasion, and the multiple positive margins, that he should receive adjuvant concurrent chemotherapy and radiation therapy to the tumor resection bed and margin as well as use intensity-modulated radiation therapy (IMRT) to track the infra-orbital nerve back to the skull base in the area of the trigeminal ganglion. He received 6,000 centigray (cGy) of a planned course of 6,600 cGy, with his course being truncated due to significant oral cavity mucositis. He received concurrent cisplatin (20 milligram per meter squared) weekly for five weeks. He was also referred to an outside institution for stereotactic body radiotherapy for the previously identified solitary lung lesion. He is disease-free two years following completion of his therapy.

\section{Discussion}

LELCS is a rare cutaneous neoplasm found most commonly in the sun-exposed skin of the head and neck although it has also been found on the trunk or extremities $[1,4]$ and is generally seen in elderly individuals [5]. Although the prognosis is generally good, there have been reports of metastases to lymph nodes at presentation [4] and local recurrence following surgical resection [1]. There is only one other case report in the literature which showed perineural invasion [6]. There has been only one reported death due to metastatic LELCS [1].

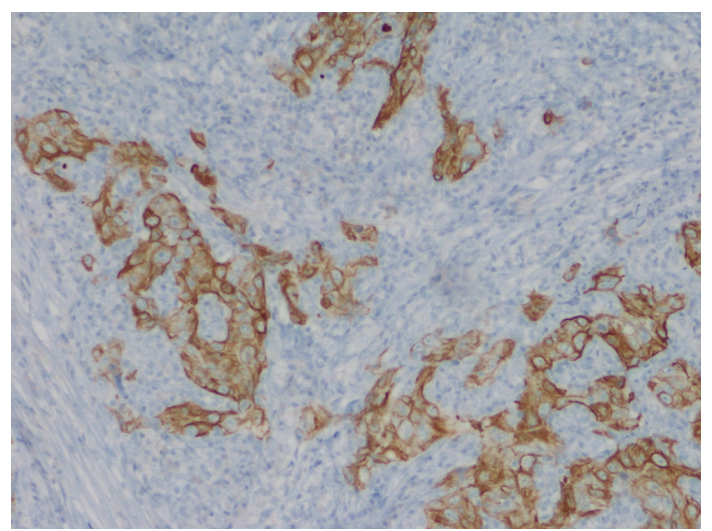

FIGURE 2: Immunohistochemical staining (100x) positive for CK$5 / 6$.

LELCS typically presents as a flesh-colored or red, firm nodule, plaque, or papule [5]. It is slow growing and generally asymptomatic and has usually been present in the patient from months to years [7]. Histologically, it is found in the mid to deep dermis and is nonecapsulated [7]. Histologically, LELCS is characterized by lobules, or nests of polygonal epithelioid cells surrounded by a dense infiltrate of T and B lymphocytes [5, 8] (Figure 1). These cytologic features are similar to those of metastatic nasopharyngeal carcinoma but are largely negative for Epstein-Barr virus (EBV) [5], although a recent case report noted an association of LELCS with EBV [9]. Immunohistochemical staining is positive for pancytokeratin and CK-5/6 (Figure 2) and p63 reactivity with the lymphoid infiltrate positive for $\mathrm{CD} 3$ and CD20 [5].

The differential diagnosis includes undifferentiated nasopharyngeal carcinoma, lymphoepithelial-like carcinoma metastasized from other sites, poorly differentiated squamous cell carcinoma, melanoma, lymphoma, and cutaneous lymphadenoma. It can generally be differentiated from undifferentiated nasopharyngeal carcinoma by the absence of EBV, notwithstanding the recent uncommon case report. It differs from squamous cell carcinoma in that it does not typically involve the overlying epidermis. Melanoma is positive for S100 and lymphoma cells will be positive for lymphoid markers. Cutaneous lymphadenoma is benign and does not have any cytological atypia.

Management should involve a complete head and neck exam to include evaluation of the nasopharynx to rule out metastasis from nasopharyngeal lymphoepithelioma [10]. Wide local excision has been the mainstay of treatment with radiation reserved for tumor recurrences $[1,2]$ or patients with lymph node metastasis at presentation [3]. There is only one other report of perineural invasion which was treated with Mohs micrographic surgery [6]. Mohs micrographic surgery has also been used as an alternative method for resecting LELCS based on the tendency of the tumor to recur following incomplete surgical excision [10, 11]. Due to the recurrent nature of the disease, the more aggressive phenotype demonstrating perineural invasion, 
and the multiple positive margins in this patient, aggressive adjuvant therapy with radiation and chemotherapy was felt to be indicated.

\section{Disclosure}

The views expressed in this paper are those of the author(s) and do not necessarily reflect the official policy or position of the Department of the Navy, Department of Defense, or the United States Government. One of the authors is a military service member and an employee of the U.S. Government. This work was prepared as part of their official duties. Title 17 U.S.C. 105 provides that "Copyright protection under this title is not available for any work of the United States Government." Title 17 U.S.C. 101 defines a United States Government work as a work prepared by a military service member or employee of the United States Government as part of that person's official duties.

\section{References}

[1] S. A. Swanson, P. H. Cooper, S. E. Mills, and M. R. Wick, "Lymphoepithelioma-like carcinoma of the skin," Modern Pathology, vol. 1, no. 5, pp. 359-365, 1988.

[2] F. J. Ortiz-Frutos, C. Zarco, R. Gil, C. Ballestin, and L. Iglesias, "Lymphoepithelioma-like carcinoma of the skin," Clinical and Experimental Dermatology, vol. 18, no. 1, pp. 83-86, 1993.

[3] S. Takayasu, M. Yoshiyama, S. Kurata, and H. Terashi, "Lymphoepithelioma-like carcinoma of the skin," Journal of Dermatology, vol. 23, no. 7, pp. 472-475, 1996.

[4] G. Hall, A. Duncan, R. Azurdia, and N. Leonard, "Lymphoepithelioma-like carcinoma of the skin: a case with lymph node metastases at presentation," American Journal of Dermatopathology, vol. 28, no. 3, pp. 211-215, 2006.

[5] P. Q. Welch, S. B. Williams, R. D. Foss, M. M. Tomaszewski, A. Gupta, and J. Ojha, "Lymphoepithelioma-like carcinoma of head and neck skin: a systematic analysis of 11 cases and review of literature," Oral Surgery, Oral Medicine, Oral Pathology, Oral Radiology and Endodontology, vol. 111, no. 1, pp. 78-86, 2011.

[6] P. Robins and M. I. Perez, "Lymphoepithelioma-like carcinoma of the skin treated by Mohs micrographic surgery," Journal of the American Academy of Dermatology, vol. 32, no. 5 I, pp. 814-816, 1995.

[7] S. Cavalieri, C. Feliciani, G. Massi et al., "Lymphoepitheliomalike carcinoma of the skin," International Journal of Immunopathology and Pharmacology, vol. 20, no. 4, pp. 851-854, 2007.

[8] N. Arsenovic, "Lymphoepithelioma-like carcinoma of the skin: new case of an exceedingly rare primary skin tumor," Dermatology Online Journal, vol. 14, no. 8, article 12, 2008.

[9] R. Aoki, H. Mitsui, K. Harada et al., "A case of lymphoepithelioma-like carcinoma of the skin associated with Epstein-Barr virus infection," Journal of the American Academy of Dermatology, vol. 62, no. 4, pp. 681-684, 2010.

[10] A. S. Glaich, D. S. Behroozan, J. L. Cohen, and L. H. Goldberg, "Lymphoepithelioma-like carcinoma of the skin: a report of two cases treated with complete microscopic margin control and review of literature," Dermatologic Surgery, vol. 32, no. 2, pp. 316-319, 2006.
[11] F. Jimenez, R. E. Clark, M. D. Buchanan, and H. Kamino, "Lymphoepithelioma-like carcinoma of the skin treated with Mohs micrographic surgery in combination with immune staining for cytokeratins," Journal of the American Academy of Dermatology, vol. 32, no. 5, pp. 878-881, 1995. 


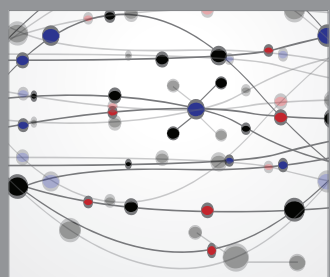

The Scientific World Journal
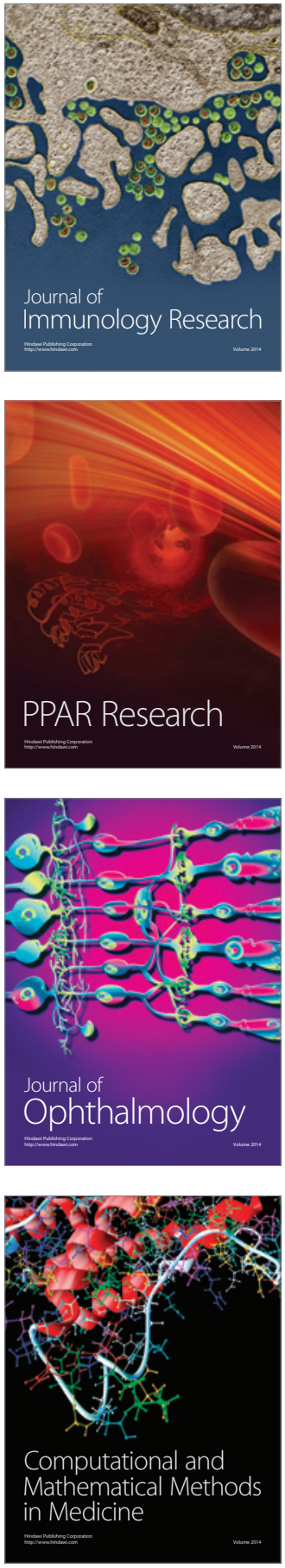

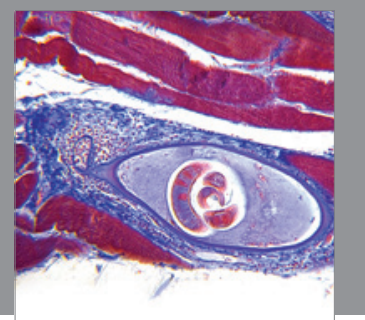

Gastroenterology

Research and Practice
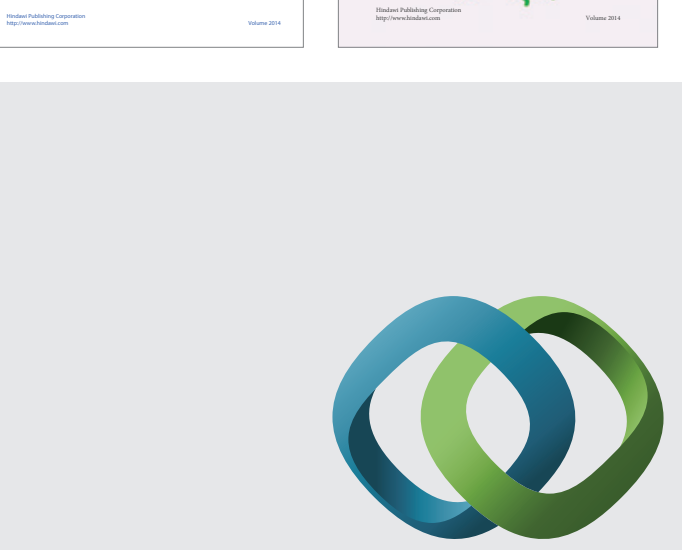

\section{Hindawi}

Submit your manuscripts at

http://www.hindawi.com
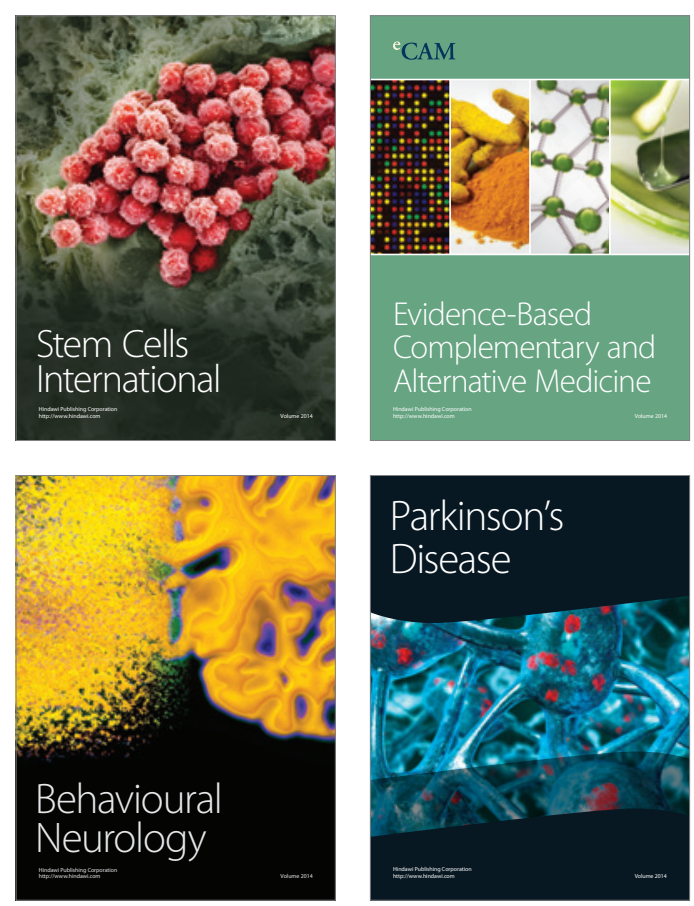

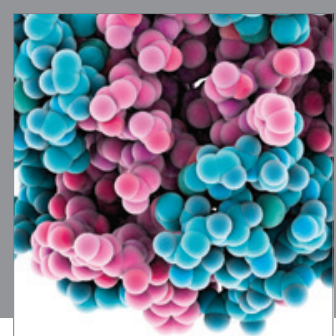

Journal of
Diabetes Research

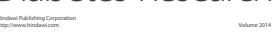

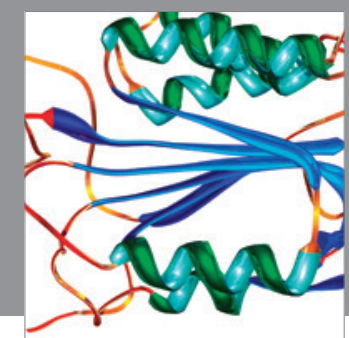

Disease Markers
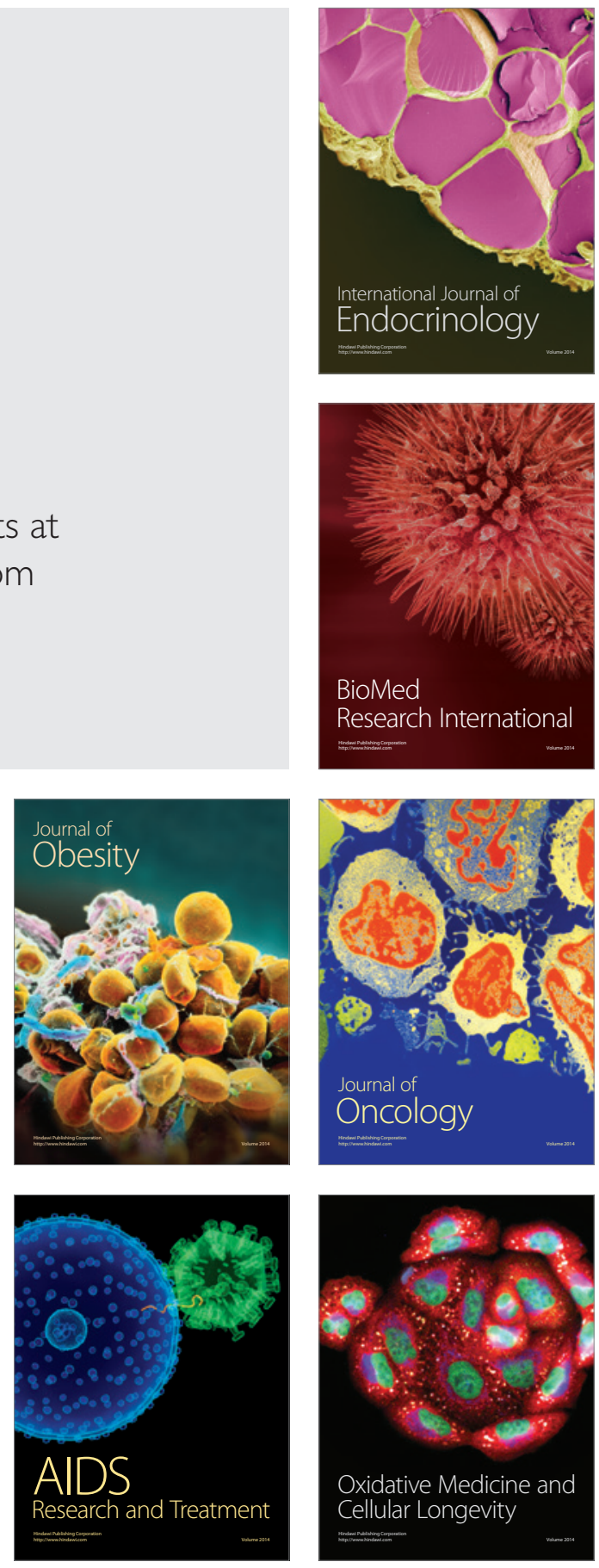